\title{
Representation of a Standard Continuous Function by a Microscope
}

Tahir H. Ismail

tahir_hsis@uomosul.edu.iq
Hind Y.Saleh

hind.saleh@uod.ac

College of Computer Sciences and Mathematics

University of Mosul, Iraq

Received on: 22/4/2010

Accepted on: 29/6/2010

\section{ABSTRACT}

The aim of this paper is to provide a representation of a standard continuous function and a standard differentiable function by mean of a microscope.

More precisely, under certain conditions, the following results have been obtained.

Let $F$ be a standard continuous function define on $\boldsymbol{R}$, and ${ }^{\circ} G$ the shadow of it's graph. If there exists a standard point $X_{0} \in \boldsymbol{R}$ and an interval $I_{0}$ about $X_{0}$ such that : $\forall X \in I_{0},(X, F(X))$ limited $\Rightarrow X \cong X_{0}$.

(i) Furthermore If there exist $X_{1}, X_{2}$ limited in $I_{0}$ such that $F\left(X_{1}\right), F\left(X_{2}\right)$ are infinitely large with opposite sign, then ${ }^{\circ} \mathrm{G}$ contains the vertical line $\Delta$ of the equation ${ }^{\circ} \mathrm{X}=X_{0}$.

(ii) If there exist a standard number $\alpha, X \in I_{0}$ and if $F(X)$ is limited such that ${ }^{\circ} F(X) \leq \alpha\left(\right.$ resp. $\left.{ }^{\circ} F(X) \geq \alpha\right)$. Also if there exist $X_{1}, X_{2}$ limited in $I_{0}$ such that $F\left(X_{1}\right)<0$ is infinitely large (resp. $F\left(X_{1}\right)>0$ ) and $F\left(X_{2}\right) \simeq \alpha$, then ${ }^{\circ} G$ contains the half line $\Delta_{\alpha}$ defined by :

$\Delta_{\alpha}=\left\{(X, Y) \in \boldsymbol{R}^{2}:^{\circ} X=X_{0},{ }^{\circ} Y \leq \alpha\left(\right.\right.$ resp. $\left.\left.{ }^{\circ} Y \geq \alpha\right)\right\}$

Let $f$ be a standard function defined at a neighborhood at a standard point $x_{0}$, then $f$ is differentiable at $x_{0}$ if and only if under every microscope of power $\varepsilon$,centered at $\left(x_{0}, f\left(x_{0}\right)\right)$, the representation of $f$ is not a vertical line at $\left(x_{0}, f\left(x_{0}\right)\right)$.

Keywords: function, standard, continuous, differentiable, microscope.

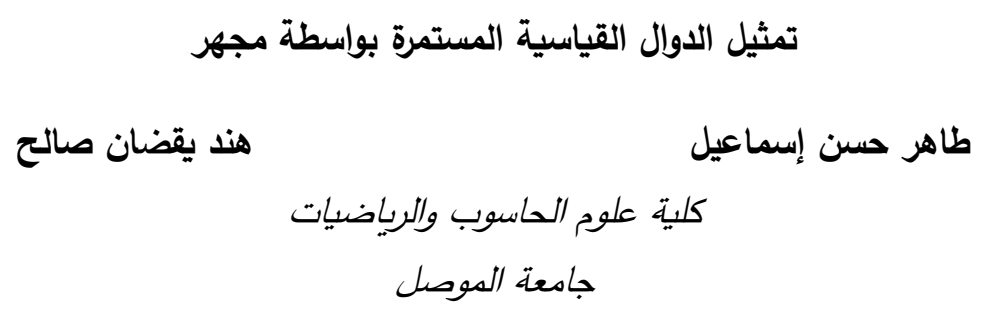

تاريخ قبول البحث: 2010/6/29

تاريخ استلام البحث:2010/4/22

\section{الملخص}

الهدف من هذا البحث هو إعطاء تمثيل للدوال القياسية المستمرة والدوال القياسية القابلة للاشتقاق باستخدام مجهر ·

بكلام أدق، وفق شروط معينة ، تم الحصول على النتائج الآتية :

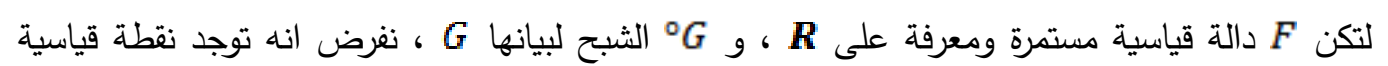

. $X \simeq X_{0} \simeq$ وفترة $X_{0} \in \boldsymbol{R}$ 


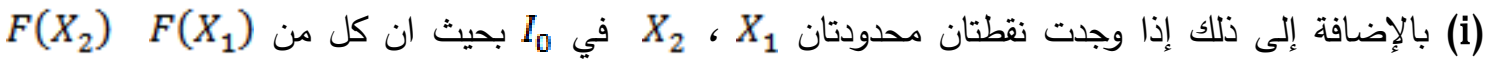
متناهية الكبر بإثارات متعاكسة ، فان Go (ii) كذلك إذا وجدت نقطتان محدودتان

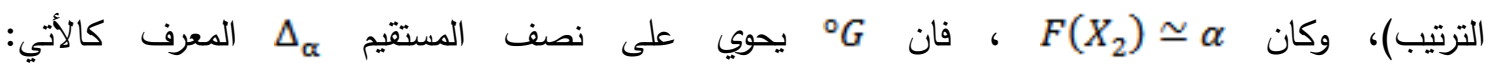

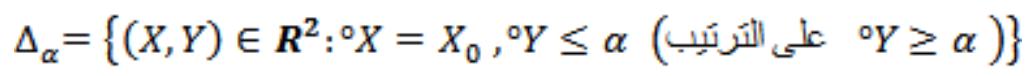

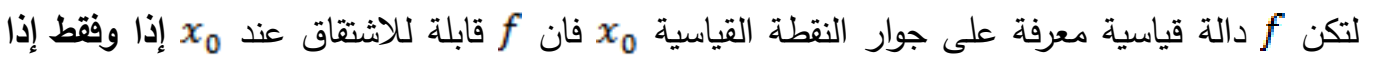
كان تحت كل مجهر بقوة ع متمركز عند النقطة (x) . $\left(x_{0}, f\left(x_{0}\right)\right)$ الكلمات المفتاحية: دالة، قياسية، مستمرة، قابلة للاشتقاق، مجهر •

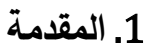

سوف نستخدم خلال هذا البحث التعاريف والرموز الآتية : التحان

إذا كان إذا كان Infinitely large إذا كان ذلك يقال أن x محدود limited .

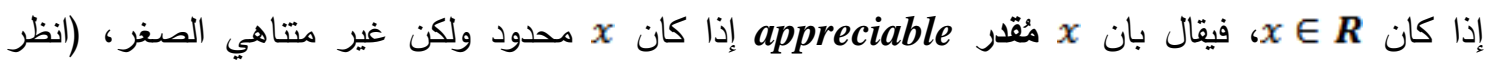
و [ذ] [12],[3],[6],[6] إذا كان x و y عنصران في R ، فيقال بان x، $x$ م متناهيتا القرب infinitely near (ويرمز لذلك y

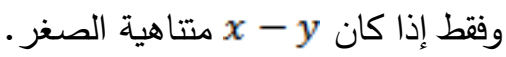
إذا كانx عنصرا في R فان مجموعة النقاط في R والتي تكون متتاهية القرب من x تسمى هالة x ويرمز لها بالرمز

إذا كانت A مجموعة جزئية من إذا كانت $A_{1}$ و $A_{2}$ مجموعتين جزئيتين من . $\left(m\left(A_{1}\right)=m\left(A_{2}\right)\right.$

إذا كانت xx نقطة محدودة في R فإنها تكون متتاهية القرب من نقطة قياسية وحيدة في R ، وان هذه النقطة

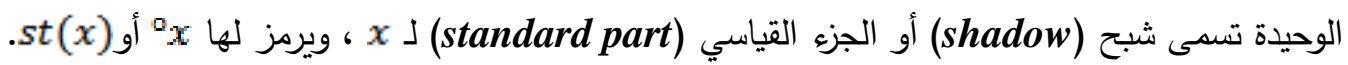
يقال لأية مجموعة معرفة في "نظرية المجموعات لزيرميلو-فرانكل مع بديهية الاختيار يرمز لها (ZFC)" بأنها

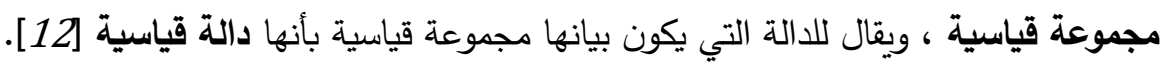

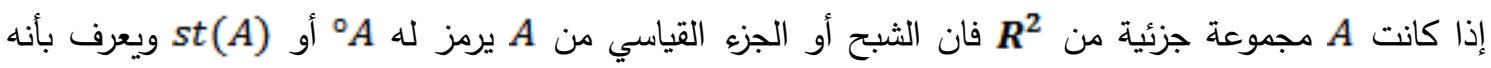

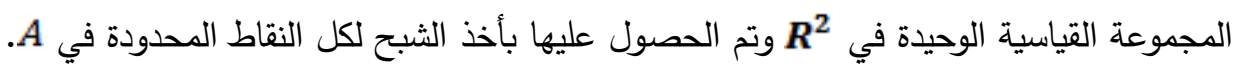
إذا لم تكن للمجموعة A نقاط محدودة فيكون شبحها A مجموعة خالية . (انظر [10],[1],[1]). لتكن f continuous ، دالة قياسية فيقال بان الدالة f مستمرة النقطة القياسية $x_{0}$ إذا كان لكل نقطتين قياسيتين 
إذا كان 0 f $\varepsilon-\operatorname{gal}\left(x_{0}\right)=\left\{x \in \boldsymbol{R} ; \frac{x-x_{0}}{\varepsilon}\right.$ هدو

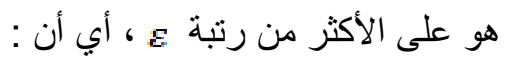
إذا كان

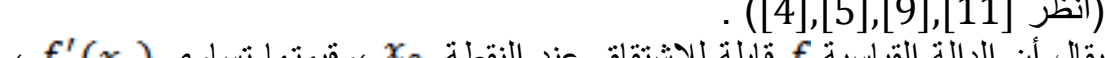
$f^{\prime}\left(x_{0}\right) \simeq \frac{f(x)-f\left(x_{0}\right)}{x-x_{0}}$

يقال أن الدالة القياسية $f$ قابلة [7]، $x \neq x_{0}, x \simeq x_{0} \quad$ لكل

بليهية الانتقال [7]: لكل صيغة قياسية تحتوي على المتغيرات المستقلة التالية تتحقق :

$$
\forall^{s t} t_{1}, t_{2}, \ldots, t_{n}\left[\forall^{s t} x F\left(x, t_{1}, t_{2}, \ldots, t_{n}\right) \Rightarrow \forall x F\left(x, t_{1}, t_{2}, \ldots, t_{n}\right)\right]
$$

$\exists x F\left(x, t_{1}, t_{2}, \ldots, t_{n}\right) \Rightarrow \exists^{s t} x F\left(x, t_{1}, t_{2}, \ldots, t_{n}\right) \quad$ وكذلك

مبرهنة القيمة المتوسطة : [8] إذا كانت f دالة مستمرة ومعرفة على الفترة [a,b]

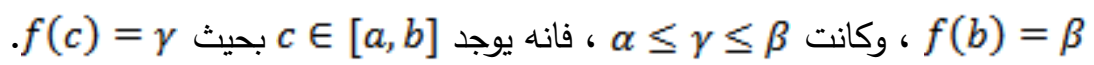

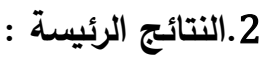

\section{أعطينا التعاريف الآتية :}

تعريف (2.1): إذا كان 0 > ع عدداً حقيقياً متناهي الصغر وكانت (x) بقوة ع والمتمركز عند النقطة $\left\{\begin{array}{l}x-x_{0}=\varepsilon X \\ y-y_{0}=\varepsilon Y\end{array}\right.$ حيث أن تعريف (2.2): يعرف تمثيل بيان دالة مستمرة f تحت مجهر بقوة ع متمركز عند نقطة (x) $F(X)=\frac{f\left(x_{0}+\varepsilon X\right)-f\left(x_{0}\right)}{\varepsilon}$ بيان دالة مستمرة F معرفة كالأتي: لبعرئ

تعريف (2.3) : لتكن f دالة قياسية مستمرة وG بيانها، فان تمثيل الدالة f تحت مجهر متمركز عند نقطة من بيانها يسمى شبح البيان G ، ويرمز له Gه تحت هذا المجهر.

المبرهنة التالية تبين لنا تمثيل الدوال القياسية القابلة الاشتقاق بواسطة مجهر

مبرهنة (2.4): لتكن f دالة قياسية قابلة للاشتقاق عند النقطة القياسية $x_{0}$ فان تمثيل الدالة f تحت كل مجهر بقوة $0>0$ محدودة ، والمستقيم $0=0$ 


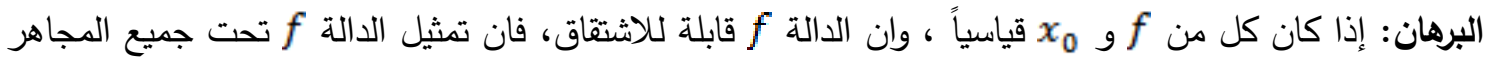
المتمركزة عند ( $)\left(x_{0} f\left(x_{0}\right)\right.$ ( لنفرض انه تحت جميع المجاهر المتمركزة عند ( ن ن

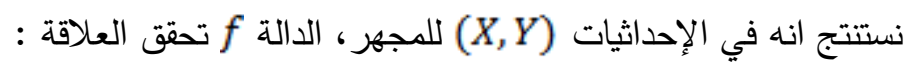
$Y \simeq a X$ لكل $\frac{Y}{X} \simeq a \quad$ لنغرض انه لكل X إذاً f و f $_{0}$ يحققان الخاصية الآتية : $\frac{f(x)-f\left(x_{0}\right)}{x-x_{0}} \simeq a$

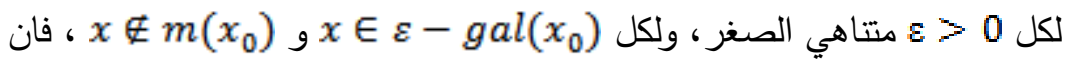

$$
\begin{aligned}
& \frac{f(x)-f\left(x_{0}\right)}{x-x_{0}} \simeq a=f^{\prime}\left(x_{0}\right) \quad \text { هذا يعني لكل } x \neq x_{0} \quad, x \simeq x_{0} \\
& Y \simeq X f^{v}\left(x_{0}\right) \text { ويتضي أن } \\
& \text { وبأخذ الثبح للطرفين نحصل على } \\
& Y \simeq X f^{\prime}\left(x_{0}\right) \text { الآن إذا كانت } \\
& \text { يقتضي إن } \\
& \text { ص. فصصل على } \\
& \text { وكتعميم للمبرهنة (2.4) لدينا المبرهنة الآتية : }
\end{aligned}
$$

مبرهنة (2.5) : لتكن f دالة قياسية معرفة على جوار النقطة القياسية $x_{0}$ فان $f$ قابلة للاشتقاق عند $x_{0}$ إذا وفقط

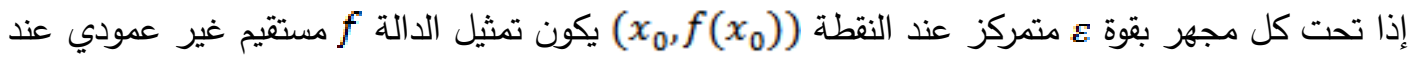
$\cdot\left(x_{0}, f\left(x_{0}\right)\right)$

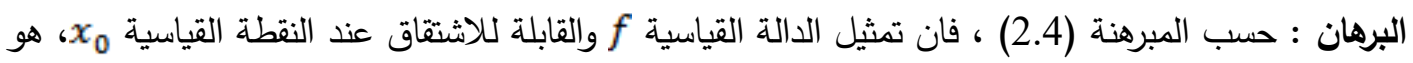
المستقيم بالعكس نفرض أن $f$ دالة قياسية ولنفرض أننا نرى المستقيم $Y=f^{\prime \prime}\left(x_{0}\right) X$ تحت كل مجهر مركزه (ذاو (او نقطة متتاهية القرب من هذا المركز

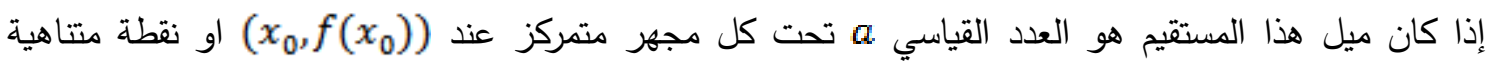
القرب منها لدينا:

$$
\begin{aligned}
& a=f^{\prime}\left(x_{0}\right) \quad \text { لكل } \\
& \frac{Y}{X} \simeq a \text { ، إذا كان لكل } \\
& \text { وعليه فان كلا من f و } x_{0} \text { تحقق الأتي: }
\end{aligned}
$$

$\frac{f(x)-f(\beta)}{x-\beta} \simeq a$

لكل $x \notin \varepsilon-m(\beta)$ ، $x \in \varepsilon-\operatorname{gal}(\beta)$ ( $x$ ، 


$$
\begin{aligned}
& \text { وهذا يعني انه لكل } \\
& \frac{f(x)-f(\beta)}{x-\beta} \simeq a \\
& \text { صسب التعريف في [7] ، فان } f \text { قابلة للاشتقاق عند } \\
& \text { مثال (2.6) : إذا كانت الدالة القياسية f معرفة كالأتي : } \\
& f(x)=\left\{\begin{array}{cl}
x^{3} \sin \frac{1}{x^{2}} & \text { if } x \neq 0 \\
0 & \text { if } x=0
\end{array}\right.
\end{aligned}
$$

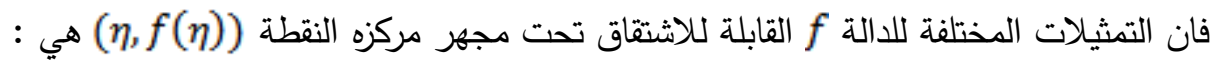

$$
\begin{aligned}
& \text { (1) مستقيم أفقي عندما } \\
& \text { (2) منحني جيبي عندما }
\end{aligned}
$$

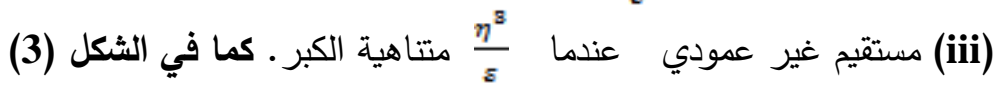

$$
\begin{aligned}
& \text { باستخدام برنامج (Matlab) حصلنا على الأشكال الآتية : }
\end{aligned}
$$

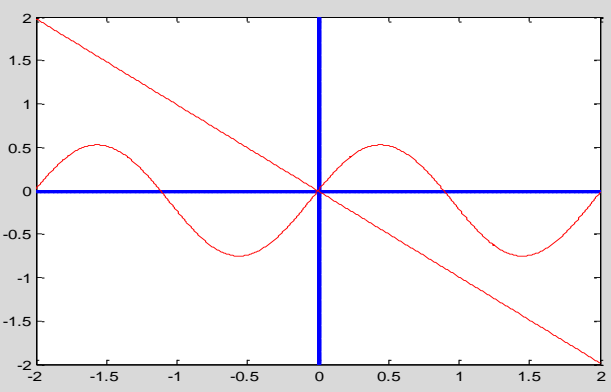

شكل (2)

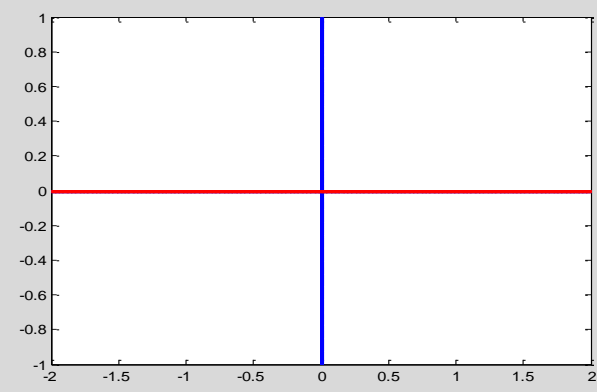

شكل (1)

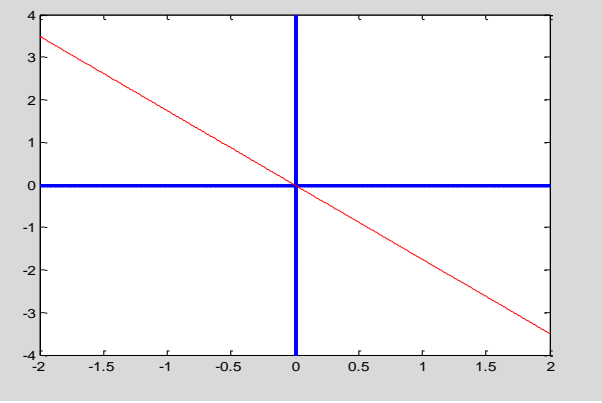

شكل (3) (l) (n)

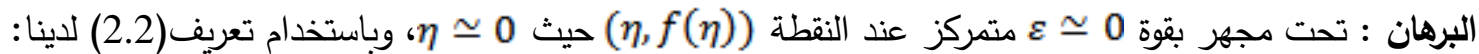
$Y=\frac{1}{\varepsilon}\left[(\varepsilon X+\eta)^{3} \sin \frac{1}{(\varepsilon X+\eta)^{2}}-\eta^{3} \sin \frac{1}{\eta^{2}}\right]$

$Y=\frac{\eta^{3}}{\varepsilon}\left[\sin \frac{1}{(\varepsilon X+\eta)^{2}}-\sin \frac{1}{\eta^{2}}\right]+\left(\varepsilon^{2} X^{3}+3 \varepsilon \eta X^{2}+3 \eta^{2} X\right) \sin \frac{1}{(\varepsilon X+\eta)^{2}}$

$Y=\frac{\eta^{3}}{\varepsilon}\left[\sin \frac{1}{(\varepsilon X+\eta)^{2}}-\sin \frac{1}{\eta^{2}}\right]$

لقيم 


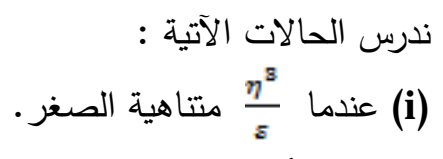

نجد أن $Y$ متتاهية الصغر لقيم X المحدودة وان التمثيل للدالة $f$ تحت هذا المجهر هو المستقيم الأفقي

$$
Y=0
$$

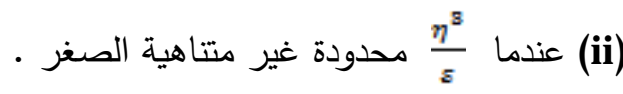
في هذه الحالة تكون $\frac{\varepsilon}{\eta}=\frac{s}{\eta^{g}} \eta^{2}$

$$
\begin{aligned}
& \sin \frac{1}{(\varepsilon X+\eta)^{2}}=\sin \frac{1}{\eta^{2}\left(\frac{\varepsilon}{\eta} X+1\right)} \\
& =\sin \frac{1}{\eta^{2}}\left(1-\frac{2 \varepsilon}{\eta} X+\delta\right) \\
& \simeq \sin \frac{1}{\eta^{2}}\left(1-\frac{2 \varepsilon}{\eta} X\right)
\end{aligned}
$$

$Y \simeq \frac{\eta^{3}}{\varepsilon}\left(\sin \left(\frac{1}{\eta^{2}}-\frac{2 \varepsilon}{\eta^{3}} X\right)-\sin \frac{1}{\eta^{2}}\right)$

عليه لقيم X المحدودة لدينا :

$Y=\frac{1}{K}\left(\sin \left(\frac{1}{\eta^{2}}-2 K X\right)-\sin \frac{1}{\eta^{2}}\right)$

نضع

$Y=\frac{1}{K}\left(\sin \frac{1}{\eta^{2}} \cos 2 K X-\cos \frac{1}{\eta^{2}} \sin 2 K X\right)-\frac{1}{K} \sin \frac{1}{\eta^{2}}$

$Y=\left(\frac{\sin \frac{1}{\eta^{2}}}{K}\right)\left(\cos \left(2^{\circ} K X\right)-1\right)-\left(\frac{\cos \frac{1}{\eta^{2}}}{K}\right) \sin \left(2^{\circ} K X\right)$

عندما (iii)

$\sin \left(\frac{1}{\eta^{2}}-2 \frac{\varepsilon}{\eta^{3}} X+\frac{\delta}{\eta^{2}}\right)=\sin \frac{1}{\eta^{2}}+\left(\frac{\delta}{\eta^{2}}-2 \frac{\varepsilon}{\eta^{3}} X\right) \cos \frac{1}{\eta^{2}}+\frac{\varepsilon^{2}}{\eta^{6}} \phi$

إذا كان X

حيث

$$
\begin{aligned}
& Y \simeq \frac{\eta^{3}}{\varepsilon}\left[\left(\frac{\delta}{\eta^{2}}-2 \frac{\varepsilon}{\eta^{3}} X\right) \cos \frac{1}{\eta^{2}}+\frac{\varepsilon^{2}}{\eta^{6}} \phi\right] \\
& Y \simeq\left(\delta \frac{\eta}{\varepsilon}-2 X\right) \cos \frac{1}{\eta^{2}}+\frac{\varepsilon}{\eta^{3}} \phi \\
& Y \simeq-2 X \cos \frac{1}{\eta^{2}}
\end{aligned}
$$

عليه لقيم X المحدودة لدينا : 
${ }^{\circ} Y=-2{ }^{\circ}\left(\cos \frac{1}{\eta^{2}}\right){ }^{\circ} \mathrm{X}$

حيث ميله (الجزء القياسي لمشتقة f عند 7 ) يأخذ كل القيم الواقعة بين 2+ و 2- .

المبرهنة التالية تبين لنا تمثيل الدوال القياسية المستمرة غير قابلة الاشتقاق بواسطة مجهر

مبرهنة (2.7) : لتكن F دالة قياسية مستمرة ومعرفة على R ، و Go الثبح لبيانها G G ، نفرض انه توجد نقطة

قياسية

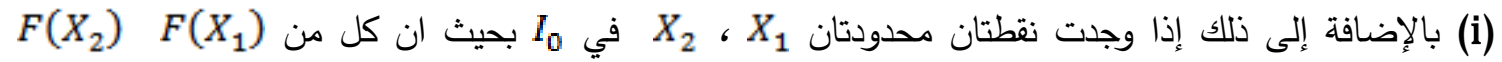
متتاهية الكبر بإثارات متعاكسة ، فان Go (ii) كذلك إذا وجدت نقطتان محدودتان على الترتيب)، وكان $\alpha$ ع

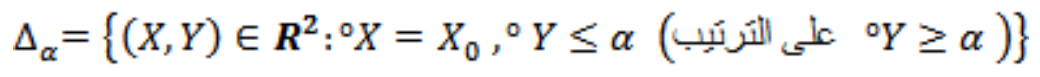

البرهان :(i) لتكن تتتميان إلى توجد $F(X)=$ Y بما أن (X) إذاً

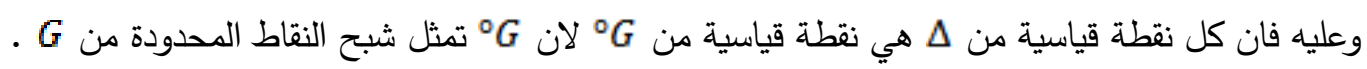

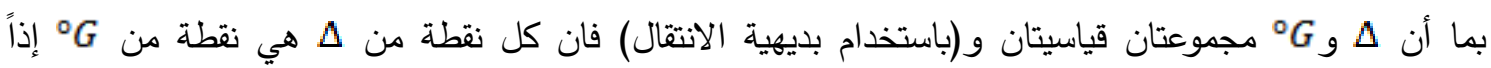

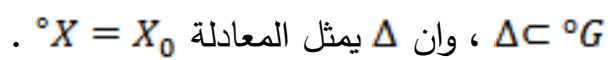

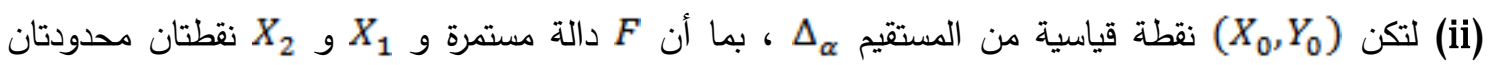
تتتميان إلى . ${ }^{\circ} F(X)=Y_{0}$ بحيث أن $X \in\left[X_{2}, X_{1}\right]$ (أو $\left.X \in X_{1}\right]$ ${ }^{\circ} F(X)=\alpha$ فندما وإذا كان وبطريقة مماثلة يمكن أن نبرهن أن

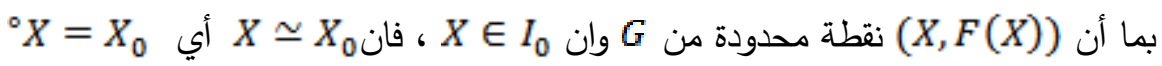
إذاً

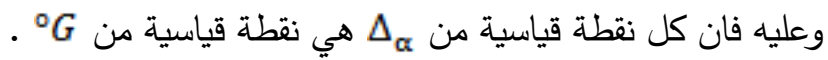

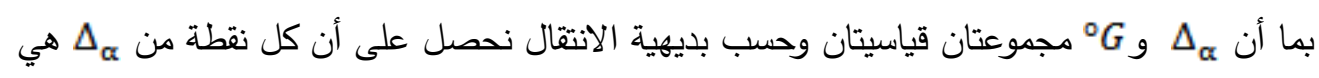


نقطة من Go.

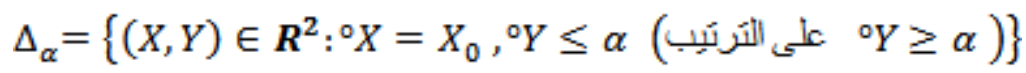

مثال (2.8) :إذا كانت $f$ دالة قياسية معرفة كالأتي : $f(x)=\sqrt{x}$ فان التمثيل للدالة f تحت مجهر بقوة : 0

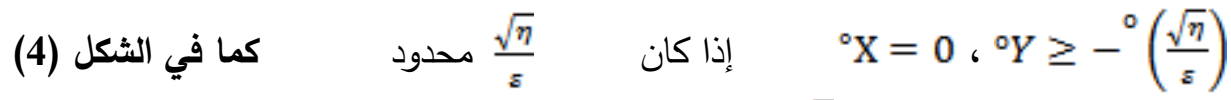

(5) $\quad{ }^{\circ} \mathrm{X}=0$

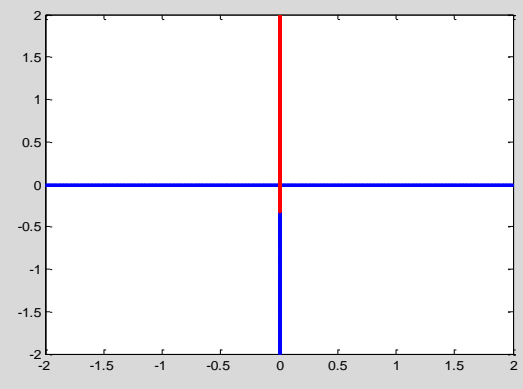

شكل (5)

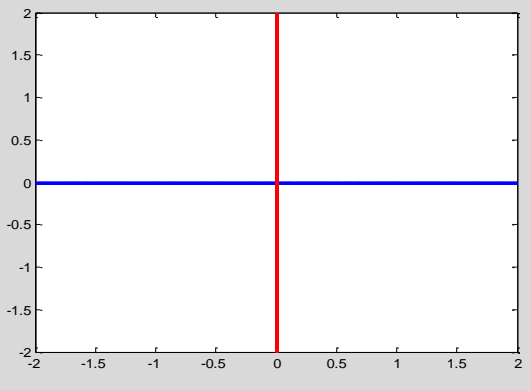

شكل (4)

البرهان : تحت مجهر بقوة ع مركزه النقطة ( $)$ (2.2) وباستخدام تعريف $Y=\sqrt{\frac{X}{s}+\frac{\eta}{s^{2}}}-\sqrt{\frac{\eta}{s^{2}}}$ نحصل على : هناك حالتان:

، عندما إذا كان عندما $L_{1}=\left\{(X, Y) \in \boldsymbol{R}^{2}:{ }^{\circ} \mathrm{X}=0,{ }^{\circ} Y \geq 0\right\}$ عندما $L_{2}=\left\{(X, Y) \in \boldsymbol{R}^{2}:^{\circ} \mathrm{X}=0,{ }^{\circ} Y \geq-^{\circ}\left(\frac{\sqrt{\eta}}{\varepsilon}\right)\right\}$ عندما

$Y=\frac{\sqrt{\eta}}{\varepsilon}\left(\sqrt{\frac{\varepsilon}{\eta} X+1}-1\right)$

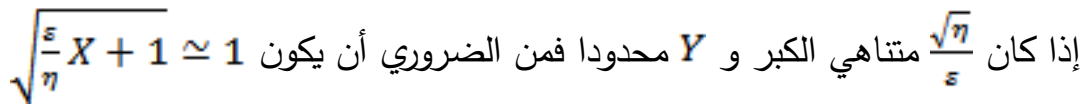
إذا كان عِ محدود غير متتاهي الصغر فمن الضروري أن تكون X متتاهية الصغر 
وإذا كان $\frac{\eta}{s}$

$\sqrt{\frac{\varepsilon}{\eta} X+1}=1+\frac{\varepsilon}{2 \eta} X+\delta$

حيث أن ס متتاهية الصغر بالنسبة إلى

$Y=\frac{\sqrt{\eta}}{\varepsilon}\left(1+\frac{\varepsilon}{2 \eta} X+\delta-1\right)$

$Y=\frac{1}{2 \sqrt{\eta}} X+\delta \frac{\sqrt{\eta}}{\varepsilon}=\frac{1}{\sqrt{\eta}}\left(\frac{X}{2}+\frac{\eta}{\varepsilon} \delta\right)$

إذا كانت النقطة (X,Y) محدودة فيكون المقدار (X) في جميع الحالات إذا كانت (X) X و $X_{1}$ ذات إثارات مختلفة.Y تأخذ القيم المتتاهية الكبر وذات إثارة متعاكسة يكون التمثيل للدالة f تحت هذا $L_{3}=\left\{(X, Y) \in R^{2}:{ }^{\circ} X=0\right\}$ المجهر هو المستقيم العمودي : 
[1] Davis M. : "Applied non standard analysis"; New- York, John Wiley and Sons (1977).

[2] Diener M. and Van Den Berg I. :"Halos and Galaxies une extention du lemme de Robinson", compte rendus de l'acadimie de science de paris . t.293 serie 1.(1983) p.385-388.

[3] Goldblatt, R. "Lectures on the Hyperreals:An Introduction to Non Standard Analysis", Springer-Verlag New York, Inc. 1998.

[4] Henle J.H. and Kleinberg E.M. :"Infinitesimal Calculus", M.I.T. Press Combridge,Mass and London ,England (1979).

[5] Ibrahim Othman Hamad, "A non standard study of the Taylor series Development", M.Sc. thesis ,University of salahaddin (2000).

[6] Ibrahim Othman Hamad,"Non standard Treatment of two dimensional Taylor series with remainder formula" , Al-Rafiden Journal of computer Sciences and Mathematics ,Mosul University ,Vol.5 ,No.1 (2005) .

[7] Nelson E. :"Internal set theory : A new approach to non standard analysis", Bull. of Amer. Math.Soc.Vol.83.No. 6 (November 1977) p.1165-1198.

[8] Pugh ,C.C., "Real Mathematical Analysis", Springer-Verlag New York, Inc. 2002.

[9] Rashad Rashid Haji, "Non standard approximation and successive shadow development ", M.Sc. thesis, University of salahaddin (2000).

[10] Robinson, A. "Non standard analysis" $2^{\text {nd }}$.ed. American Elsevier New-York (1970).

[11] Stroyan K.D. and Luxemburg W.A., "Introduction to the theory of infinitesimal ,New-York, Academic press (1976).

[12] Vladimir, K. and Reeken, M. "Non standard Analysis , Axiomatically" ,Springer-Verlag Berlin Heidelberg 2004. 